\title{
BUCKLING ANALYSIS OF GRID-STIFFENED COMPOSITE SHELLS
}

\author{
Dan WANG and Mostafa ABDALLA \\ Delft University of Technology, 2629 HS Delft, The Netherlands \\ e-mail: d.wang@tudelft.nl,m.m.abdalla@tudelft.nl
}

Key words: Buckling, Grid-Stiffened Structures, Composite, Homogenization Techniques, Bloch Wave Theory

\begin{abstract}
There is a renewed interest in grid-stiffened composite structures; they are not only competitive with conventional stiffened constructions and sandwich shells in terms of weight but also enjoy superior damage tolerance properties. In this paper, both global and local structural instabilities are investigated for grid-stiffened composite panels using homogenization theory. Characteristic cell configurations with periodic boundary constraints are employed for orthogrid- and isogrid-stiffened shells in order to smear the stiffened panel into an equivalent unstiffened shell. Homogenized properties corresponding to classical lamination theory are obtained by matching the strain energy of the stiffened and equivalent cells. Global buckling analysis is carried out based on the homogenized shell properties. Bloch wave theory is adopted to calculate the local buckling load of grid-stiffened shells, where the interaction of adjacent cells is fully taken into account. Moreover, instead of considering skin buckling and stiffener crippling separately, as is commonly done, the skin and stiffeners are assembled together at the level of the characteristic cell. The critical instabilities can be captured whether they are related to the skin or stiffener or their interaction. The proposed combination of global/local models can also be used to predict the material failure of a structure. Numerical examples of orthogrid- and isogrid-stiffened isotropic panels show that the local buckling loads predicted by the proposed method match finite element calculations better than semi-analytical methods based on assumptions and idealisations. The proposed method is further validated using typical configurations of flat composite panels and circular composite cylinders.
\end{abstract}

\section{Introduction}

Due to the advantages of lightweight, small manufacturing cost, high strength, high stability, great energy absorption and superior damage tolerance $[1,2,3,4]$, grid-stiffened composite panels have been applied in aerospace engineering for payload fairings of launch vehicles and for load-bearing structures of satellites. Usually, three basic types of grids, i.e., isogrid, orthogrid, and angle-grid, as shown in Fig. 1, are used in practical applications. 


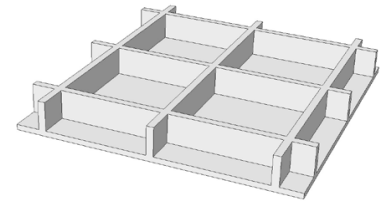

(a) Orthogrid

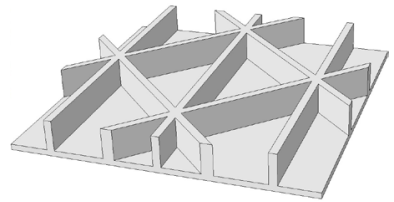

(b) Isogrid

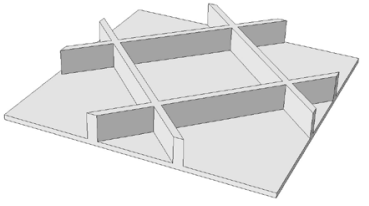

(c) Anglegrid

Figure 1: Basic types of grid-stiffened panels.

Many researchers have devoted themselves to studying grid-stiffened composite panels [5, $6,7,8,9]$. The discrete stiffener and the smeared stiffener methods are two main methods used to study behaviour of such structures. With the detailed geometry retained, the discrete stiffener method, where the skin and stiffeners are modeled separately with the compatibility maintained at the interface [7], has the ability to capture both global and local behaviour and guarantee high accuracy [8]. However, using the detailed geometry is not only computationally expensive for large structures but also precludes the application of the discrete method in downstream design optimization due to the difficulty of automatic remeshing for changeable geometry. Instead, the smeared stiffener method, where a grid-stiffened structure is represented by an equivalent homogeneous panel, only involves simple geometry without any detailed geometric information and therefore leads to better computational efficiency and provides a flexible and suitable interface with design optimization. However, as proved by researchers [8], the smeared stiffener method is only accurate for calculating global buckling and deficient for local buckling.

Homogenization theory has been widely used in calculating effective properties of a heterogeneous medium. By using asymptotic expansions and the assumption of periodicity, physical quantities can be evaluated on two different levels: the macroscopic and microscopic, where the former implies slow variation and the latter implies rapid oscillations [10]. Two different families of numerical methods based on Finite Element Method and Fast Fourier Transform$\mathrm{s}$, respectively, were compared to obtain the effective properties of composites with periodic microstructure [11]. In this work, two specific features of periodic homogenization including periodicity conditions and strain or stress control were presented in details. As an extension of a homogeneous Love-Kirchhoff model [12], a homogenized Reissner-Mindlin model with the shear effects of thick plates taken into account was proposed, where the shear constants were determined by an auxiliary 3D boundary value problem [13]. Using the full gradient of the bending moment instead of only the mixed shear forces, the Bending-Gradient theory $[14,15]$ took advantage of the higher accuracy of both deflection and local stresses than the ReissnerMindlin theory for heterogeneous plates and was further applied to a folded core sandwich panel [16] and a periodic beam lattice [17].

Grid-stiffened panels may fail in three different modes of rib/stiffener crippling, skin buckling and global buckling, as show in Fig. 2, where the former two are known as local buckling. To increase its service life, a grid-stiffened structure is usually designed to localize buckling to ensure that global buckling happens after local buckling. Practically, global buckling loads of grid-stiffened panels are usually assessed using the smeared method, while local buckling 
loads are predicted approximately with boundary conditions properly assumed at the interface of skin and stiffeners. The global buckling load for a axial compressed cylinder was obtained in a closed form for classical simply supported ends, while for the case of a combined axial compression and torsion, the global buckling problem was formulated using the Galerkin approach [6]. Moreover, with the assumption of a simple support at the stiffener/skin attachment, the skin buckling was calculated with the skin cell simply supported along its edges and the stiffener crippling was calculated with a clamped fixity condition imposed at the nodes. Weber and Middendorf [18] integrated the interaction between adjacent skin fields into the calculation of the local skin buckling load by applying periodic boundary conditions at opposite panel edges. Both global and local buckling behaviours were considered in a weight-minimum design for grid-stiffened panels and cylinders using a genetic algorithm $[19,20]$. In the work, an improved smeared theory with the offset effect of stiffeners included [21] was employed for the global analysis, while the local skin buckling was assessed by the Rayleigh-Ritz method which accounts for material anisotropy and stiffener crippling was assessed using the method provided in Reddy's work [6]. Then, this method was applied to optimization design of grid-stiffened panels with variable curvature, which were modeled as assemblies of panels with constant curvature [22]. To carry out an optimization design for aircraft wing rib panels, global bucking, stiffener crippling and material failure were evaluated using the Lagrange multiplier method, the assumption that the stiffener blade was an axially loaded rectangular plate and a maximum strain failure criterion, respectively [23]. A hybrid model was proposed [24] with elements around the loading node of a smeared panel replaced by the detailed rib and skin elements to obtain a refined stress analysis, which could also take hygrothermal behaviour and local buckling into account.
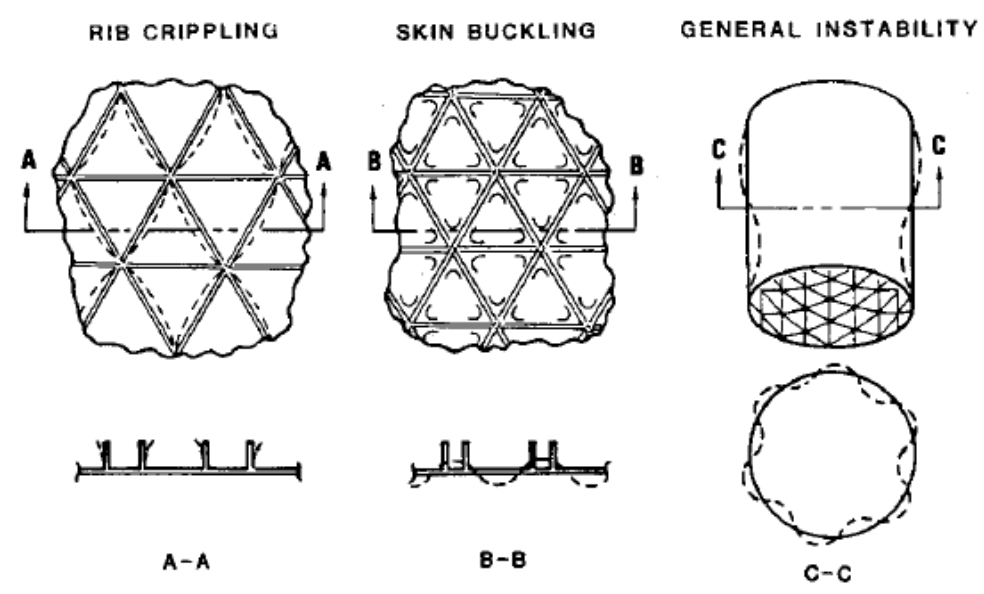

Figure 2: Buckling modes of grid-stiffened panels. [6]

A different approach to calculate local instability in periodic media is furnished by Bloch's wave theory. In the Bloch wave theory, the displacement of a particle in a periodic medium could be represented as a product of a periodic component with the same periodicity as the 
medium and periodic function with an arbitrary wavelength. According to this special representation, wave propagation in periodic beams and stiffened plates was evaluated $[25,26]$. Then, it was proved that the Bloch wave theory had the ability to determine the onset of instability in periodic solids [27]. A great body of related work was done by Triantafyllidis and co-workers $[4,28,29,30,31]$ to investigate critical material instability in periodic solids. In their work, concepts of a micro-failure surface and a macro-failure surface were introduced to describe the locus of first microscopic bifurcation points and points with a macroscopic loss of ellipticity, respectively. The distinction between onsets of microscopic and macroscopic material instability is whether the wavelength is commensurate with the unit cell dimension or not. Research has showed that the micro-failure surface of an infinite perfectly periodic structure is a lower bound of that of a finite structure, and an upper bound of that of a finite structure with imperfections [30]. Applications of the Bloch wave theory in two-dimensional periodic composites showed that the interstitial stiffness had an important influence on the nature of the critical failure mode [28]. Similar work has been done by Ohno and co-workers [32, 33] to establish the critical states for square honeycombs and Kelvin cell foams under compression loadings.

As clearly illustrated in references $[34,35]$, existing methods based on the Bloch wave theory focus on material failure where the material deforms inside the plane as a failure mode. In this paper, the out-of-plane instability of grid-stiffened panels is characterized by the Bloch wave theory. In contrast to the existing methods used to detect material failure, only local instability can be investigated in this case, while global instability is evaluated using the equivalent unstiffened panel with average material properties.

The rest of this paper is devoted to a description of the homogenization approach and the use of Bloch's wave theory in detecting local instability of grid-stiffened structure. Section 3 presents an overview of the homogenization approach and the smearing process of a gridstiffened panel. Section 4 develops the Bloch wave theory and reduces the computation to a symmetric generalized eigenvalue problem. Section 5 presents a number of numerical examples of flat and curved grid-stiffened structures and comparison to published results and detailed finite element calculations. Finally, section 6 provides conclusions.

\section{Homogenization of a grid-stiffened composite panel}

\subsection{Homogenization theory}

According to the homogenization theory for media with periodic structures [10], the gridstiffened panel can be investigated using a double-scale scheme. From the macroscopic perspective, the whole structure can be treated as a homogenous panel without any stiffener. Displacements are assumed to be smooth and change slowly over one cell from the macroscopic perspective. From the microscopic perspective, the displacement will have a significant, but smaller amplitude, variation over one cell. Therefore, the displacement can be represented in the following expression as a function of two scales. For the out-of-plane deflection we have:

$$
w(\mathbf{x})=w_{0}(\mathbf{x})+\eta w_{1}(\mathbf{x}, \mathbf{y})+\eta^{2} w_{2}(\mathbf{x}, \mathbf{y})+\ldots
$$


where $\mathbf{y}=\mathbf{x} / \eta$, and $\eta$ is a small parameter representing the ratio of cell dimension to the dimension of the solid.

Correspondingly, the curvature vector can be expressed as:

$$
w_{, \mathbf{x x}}(\mathbf{x})=\frac{1}{\eta} w_{1, \mathbf{y y}}(\mathbf{x}, \mathbf{y})+\left(w_{0, \mathbf{x x}}(\mathbf{x})+w_{2, \mathbf{y y}}(\mathbf{x}, \mathbf{y})\right)+\eta\left(w_{1, \mathbf{x} x}(\mathbf{x}, \mathbf{y})+w_{3, \mathbf{y} \mathbf{y}}(\mathbf{x}, \mathbf{y})\right)+\ldots
$$

For the above equation, if $w_{1, \mathbf{y y}} \neq \mathbf{0}$, the first item in the right part of that equation will become unbounded as $\eta \rightarrow 0$. Therefore, we require $w_{1}=0$ and Eq. (2) can be rewritten as:

$$
\boldsymbol{\kappa}(\mathbf{x})=\boldsymbol{\kappa}_{0}(\mathbf{x})+\boldsymbol{\kappa}_{1}(\mathbf{x}, \mathbf{y})+\eta \boldsymbol{\kappa}_{2}(\mathbf{x}, \mathbf{y})+\ldots
$$

where $\boldsymbol{\kappa}_{0}$ is the curvature due to the macroscopic variation and $\boldsymbol{\kappa}_{1}$ due to the microscopic variation. Higher order terms will not be considered in this work.

Similarly, the in-plane displacement is represented as:

$$
\mathbf{u}(\mathbf{x})=\mathbf{u}_{0}(\mathbf{x})+\eta \mathbf{u}_{1}(\mathbf{x}, \mathbf{y})+\eta^{2} \mathbf{u}_{2}(\mathbf{x}, \mathbf{y})+\ldots
$$

and the corresponding strains:

$$
\boldsymbol{\varepsilon}(\mathbf{x})=\boldsymbol{\varepsilon}_{0}(\mathbf{x})+\boldsymbol{\varepsilon}_{1}(\mathbf{x}, \mathbf{y})+\eta \boldsymbol{\varepsilon}_{2}(\mathbf{x}, \mathbf{y})+\ldots
$$

The displacement fields $w_{2}(\mathbf{x}, \mathbf{y})$ and $\mathbf{u}_{1}(\mathbf{x}, \mathbf{y})$ are assumed to be periodic in $\mathbf{y}$.

\subsection{Smearing based on the conservation of the strain energy}

In the smeared stiffener method, a grid-stiffened panel is replaced with an equivalent homogeneous panel with uniform properties. The homogenization process is performed by equating the strain energy of the equivalent model to be the same as that of the detailed cell configuration.

For simplicity, consider a cell configuration periodic along the $y_{1}$ and $y_{2}$ directions. The cell average in-plane strains are given by:

$$
\left\{\begin{array}{l}
\varepsilon_{01}=u_{01, y_{1}} \\
\varepsilon_{02}=u_{02, y_{2}} \\
\gamma_{01 y_{2}}=u_{01, y_{2}}+u_{02, y_{1}}
\end{array}\right.
$$

Therefore, these displacements can be represented analytically.

$$
\left\{\begin{array}{l}
u_{01}=\varepsilon_{01} y_{1}+\frac{1}{2} \gamma_{012} y_{2}+c_{1} \\
u_{02}=\varepsilon_{02} y_{2}+\frac{1}{2} \gamma_{012} y_{1}+c_{2}
\end{array}\right.
$$

Similarly, following compatible relations hold between average curvatures and the corresponding deflection $u_{03}$ : 


$$
\left\{\begin{array}{l}
\kappa_{01}=u_{03, y_{1} y_{1}} \\
\kappa_{02}=u_{03, y_{2} y_{2}} \\
\kappa_{012}=2 u_{03, y_{1} y_{2}}
\end{array}\right.
$$

The slowly varying out-of-plane deflection may be represented as follows:

$$
\begin{aligned}
u_{03} & =\frac{1}{2}\left\{\begin{array}{ll}
y_{1} & y_{2}
\end{array}\right\}\left[\begin{array}{cc}
\kappa_{01} & \kappa_{012} / 2 \\
\kappa_{012} / 2 & \kappa_{02}
\end{array}\right]\left\{\begin{array}{l}
y_{1} \\
y_{2}
\end{array}\right\}+c_{3} y_{1}+c_{4} y_{2}+c_{5} \\
& =\frac{1}{2}\left(\kappa_{01} y_{1}^{2}+\kappa_{02} y_{2}^{2}+\kappa_{012} y_{1} y_{2}\right)+c_{3} y_{1}+c_{4} y_{2}+c_{5}
\end{aligned}
$$

In the above expressions of Eqs. (7) and (9), items including constants $c_{1}, c_{2}$ and $c_{5}$ denote rigid body translations and items including constants $c_{3}$ and $c_{4}$ denote rigid body rotations, which have no contribution to the strain energy. Therefore, all these constants could be set to zero to simplify the expressions.

The rotations $\theta_{01}=u_{03, y_{2}}, \theta_{02}=-u_{03, y_{1}}$ are given by:

$$
\left\{\begin{array}{l}
\theta_{01}=\kappa_{02} y_{2}+\frac{1}{2} \kappa_{012} y_{1} \\
\theta_{02}=-\kappa_{01} y_{1}-\frac{1}{2} \kappa_{012} y_{2}
\end{array}\right.
$$

The periodic part of the displacement vector does not contribute to the difference in total displacement between corresponding nodes on opposite sides of a characteristic cell. Thus the following boundary conditions apply:

$$
\left\{\begin{aligned}
\Delta u_{1} & =\varepsilon_{01} \Delta y_{1}+\frac{1}{2} \gamma_{012} \Delta y_{2} \\
\Delta u_{2} & =\varepsilon_{02} \Delta y_{2}+\frac{1}{2} \gamma_{012} \Delta y_{1} \\
\Delta u_{3} & =\frac{1}{2} \kappa_{012} \Delta\left(y_{1} y_{2}\right) \\
\Delta \theta_{1} & =\kappa_{02} \Delta y_{2}+\frac{1}{2} \kappa_{012} \Delta y_{1} \\
\Delta \theta_{2} & =-\kappa_{01} \Delta y_{1}-\frac{1}{2} \kappa_{012} \Delta y_{2}
\end{aligned}\right.
$$

Herein, the center of the cell is set to the origin of the coordinate system to simplify the equations.

The total strain energy is given by:

$$
\mathscr{U}=\frac{1}{2} \int_{\Omega_{c}} \Gamma_{0}^{T} \mathbf{C} \Gamma_{0} d \Omega_{c}
$$

Here, $\Gamma_{0}=\left\{\boldsymbol{\varepsilon}_{\mathbf{0}} \boldsymbol{\kappa}_{\mathbf{0}}\right\}^{T}$ and symbol $\mathbf{C}$ denotes the equivalent material stiffness matrix of the smeared panel. 
The discrete version takes the form:

$$
\mathscr{U}=\frac{1}{2} \mathbf{u}^{T} \mathbf{K u}
$$

By rewriting the displacement constraints of Eq. (11) in a matrix form $\mathbf{L u}=\mathbf{D} \Gamma_{0}$, we can develop an augmented form of the potential energy for a system without any external force.

$$
\Pi=\frac{1}{2} \mathbf{u}^{T} \mathbf{K u}-\lambda^{T}\left(\mathbf{L u}-\mathbf{D} \Gamma_{0}\right)
$$

Using the minimum potential energy principle, we have:

$$
\left\{\begin{array}{l}
\frac{\partial \Pi}{\partial \mathbf{u}}=\mathbf{K u}-\mathbf{L}^{T} \lambda=0 \\
\frac{\partial \Pi}{\partial \lambda}=\mathbf{L u}-\mathbf{D} \Gamma_{0}=0
\end{array}\right.
$$

which leads to

$$
\left[\begin{array}{cc}
\mathbf{K} & \mathbf{L}^{T} \\
\mathbf{L} & \mathbf{0}
\end{array}\right]\left\{\begin{array}{l}
\mathbf{u} \\
\lambda
\end{array}\right\}=\left\{\begin{array}{l}
\mathbf{0} \\
\mathbf{D}
\end{array}\right\} \Gamma_{0}
$$

Solving the above system with imposing strains and curvatures evaluated in the macroscopic level as 'generalized loading' [11], displacements in the local region are obtained. Then, local stresses, which determines the material failure, can be acquired.

The solution of Eq. (16) takes the form of $\mathbf{u}=\mathbf{U} \Gamma_{0}$. With a combination of Eq.s (12) and (13) and a substitution of elementary strain and curvature states expressed by an unitary matrix into Eq. (12), the equivalent stiffness matrix of the smeared panel can be obtained via the following expression.

$$
\mathbf{C}_{e f f}=\frac{1}{A_{c}} \mathbf{U}^{T} \mathbf{K U}
$$

\section{Local buckling prediction}

For a characteristic cell of a grid-stiffened panel, the equilibrium of its finite element model could be expressed as follows:

$$
\left[\begin{array}{ccc}
\mathbf{K}_{1,1}^{t} & \mathbf{K}_{1,2:(n-1)}^{t} & \mathbf{K}_{1, n}^{t} \\
\mathbf{K}_{2:(n-1), 1}^{t} & \mathbf{K}_{2:(n-1), 2:(n-1)}^{t} & \mathbf{K}_{2:(n-1), n}^{t} \\
\mathbf{K}_{n, 1}^{t} & \mathbf{K}_{2:(n-1), n}^{t} & \mathbf{K}_{n, n}^{t}
\end{array}\right]\left\{\begin{array}{c}
\mathbf{u}_{1} \\
\mathbf{u}_{2:(n-1)} \\
\mathbf{u}_{n}
\end{array}\right\}=\left\{\begin{array}{c}
\mathbf{f}_{1} \\
\mathbf{0} \\
\mathbf{f}_{n}
\end{array}\right\}
$$

where $\mathbf{K}^{t}$ is the tangent stiffness matrix. $\mathbf{u}$ and $\mathbf{f}$ are the displacement and force vectors, respectively. Subscripts 1 and $n$ denote the starting part and the ending part of the periodic cell along the direction of the wave propagation, respectively. 
According to the Bloch wave theory, displacement vectors of a point in a $2 \mathrm{D}$ periodic structure coulcand be expressed in the following form [30]:

$$
\mathbf{u}\left(y_{1}, y_{2}\right)=\tilde{\mathbf{u}}\left(y_{1}, y_{2}\right) \exp \left[i\left(\frac{m_{1} y_{1}}{s_{1}}+\frac{m_{2} y_{2}}{s_{2}}\right)\right]
$$

where $m_{1}$ and $m_{2}$ are dimensionless wave numbers and $s_{1}$ and $s_{2}$ are the cell lengths along two different periodic axes. $\tilde{\mathbf{u}}\left(y_{1}, y_{2}\right)$ is a periodic function following the same cycle of a representative cell, which satisfies:

$$
\tilde{\mathbf{u}}\left(y_{1}+n_{1} s_{1}, y_{2}+n_{2} s_{2}\right)=\tilde{\mathbf{u}}\left(y_{1}, y_{2}\right)
$$

In the above equation, $n_{1}$ and $n_{2}$ are arbitrary integers.

The representation of $\mathbf{u}$ in Eq. (19) implies the following relationship at the periodic boundaries:

$$
\mathbf{u}_{n}=\boldsymbol{\mu}\left(e^{i m_{1}}, e^{i m_{2}}\right) \mathbf{u}_{1}
$$

Construct a matrix as follows:

$$
\mathbf{L}=\left\{\begin{array}{lll}
-\overline{\boldsymbol{\mu}} & \mathbf{0} & \mathbf{I}
\end{array}\right\}^{T}
$$

with $\overline{\boldsymbol{\mu}}$ denotes the conjugate matrix of $\boldsymbol{\mu}$. Note that the matrix $\mathbf{L}$ is identical to that used in the smearing process when the wave numbers equal to zero. Then, the conjugate transpose matrix of $\mathbf{L}$ could be expressed as:

$$
\mathbf{L}^{*}=\left\{\begin{array}{lll}
-\boldsymbol{\mu} & \mathbf{0} & \mathbf{I}
\end{array}\right\}
$$

It is easy to conclude that:

$$
\mathbf{L}^{*} \mathbf{u}=\mathbf{0}
$$

The forces between boundary nodes on the opposite edges obey the same relationship as the displacements expressed by Eq. (21) but with opposite directions. Therefore, the following equation is satisfied.

$$
\mathbf{f}=\mathbf{L f}_{n}
$$

With the substitution of Eqs. (24) and (25), Eq. (18) could be rewritten as [31]:

$$
\left[\begin{array}{ll}
\mathbf{K}^{t} & \mathbf{L} \\
\mathbf{L}^{*} & \mathbf{0}
\end{array}\right]\left\{\begin{array}{c}
\mathbf{u} \\
-\mathbf{f}_{n}
\end{array}\right\}=\mathbf{0}
$$

The generalized eigenvalues of the above equation are the buckling loads of the periodic panel. The minimum eigenvalue $\Lambda_{m}\left(m_{1}, m_{2}\right)$ for prescribed dimensionless wave numbers from 
$2 n \pi$ to $(2 n+2) \pi$ defines the critical buckling load parameter surface, where $n$ denotes an nonnegative integer. Due to the periodicity and symmetry, the range of $m_{1}$ and $m_{2}$ can be limited to $[0, \pi]$. Therefore, the critical buckling load defined as the minimum value of all the $\Lambda_{m}\left(m_{1}, m_{2}\right)$ satisfies the following equation [30].

$$
\Lambda_{c}=\min _{0 \leq m_{1}, m_{2} \leq \pi} \Lambda_{m}\left(m_{1}, m_{2}\right)
$$

Actually, in contrast to periodic solid, a grid-stiffened thin-walled panel with infinite dimensions always buckles at zero, which belongs to global instability with a long wavelength. In this case, the local minimum value of Eq. (27) with the wave numbers far away from zero belongs to the range of a short wavelength, which is the local critical buckling load for a finite panel. The local buckling load of a finite grid-stiffened composite panel is obtained within this framework.

\section{Numerical examples}

In this section, numerical examples are used to demonstrate the effectiveness of the proposed approach. First, the local buckling loads of an isotropic simply supported panel predicted by the proposed method are compared with those by a semi-analytic method [18]. Then, numerical predictions of the proposed method are presented for typical orthogrid- and isogrid-stiffened composite flat panels and circular cylinders. For the examples, detailed finite element models provide the basis for validating the accuracy of the proposed method.

\subsection{A flat isotropic panel}

A comparison between local buckling loads of the proposed method and a semi-analytical skin buckling calculation method [18] is carried out in the example for orthogrid- and isogridstiffened isotropic panels under axial compression. In Weber and Middendorf's work, buckling load coefficients of both single panels with simply supported boundary conditions and skin fields with periodic boundary conditions imposed at opposite panel edges are provided. Except for the case of the rectangular panel under compression, there are evident differences between the results predicted by these two different assumptions. The results of both cases are considered in the comparison. The semi-analytical buckling results of the rectangular panel with clamped boundary conditions [36] are also included.

The different cell configurations involved for orthogrid and isogrid are illustrated in Fig.s 3(a) and (b), respectively. To eliminate the effect of dimensions and material properties, a dimensionless buckling load coefficient $k$ is used with an expression as follows:

$$
k=\frac{N_{c r} b^{2}}{\pi^{2} D}
$$

where

$$
D=\frac{t^{3} E}{12\left(1-v^{2}\right)}
$$


(a)

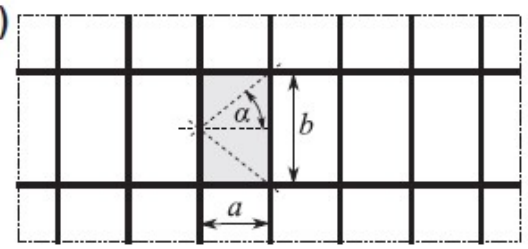

(b)

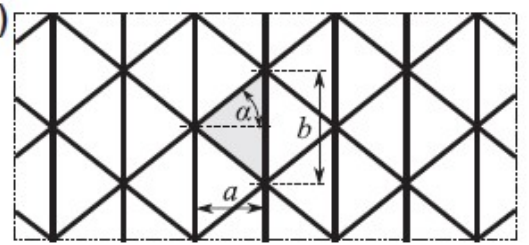

Figure 3: Sketch of characteristic cell configurations of an isotropic panel [18].

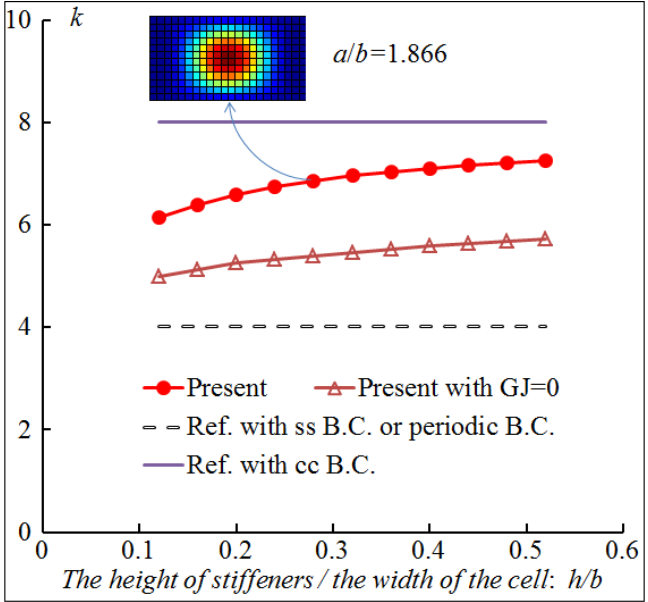

(a) $a / b=1.866$

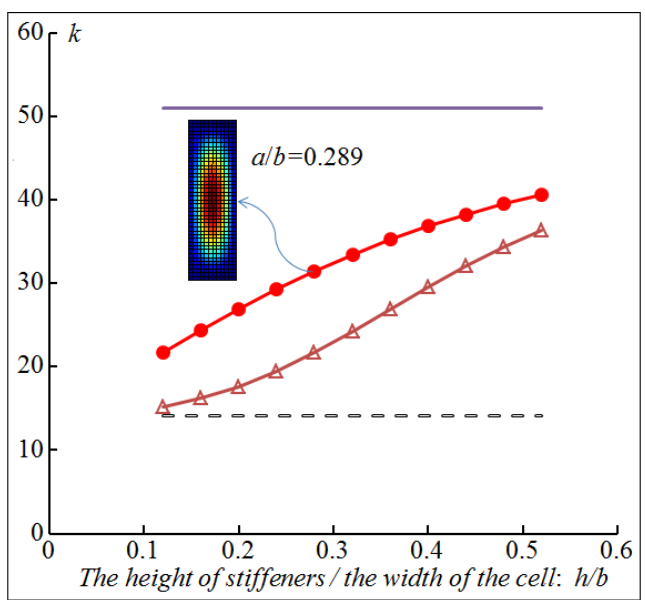

(c) $a / b=0.289$

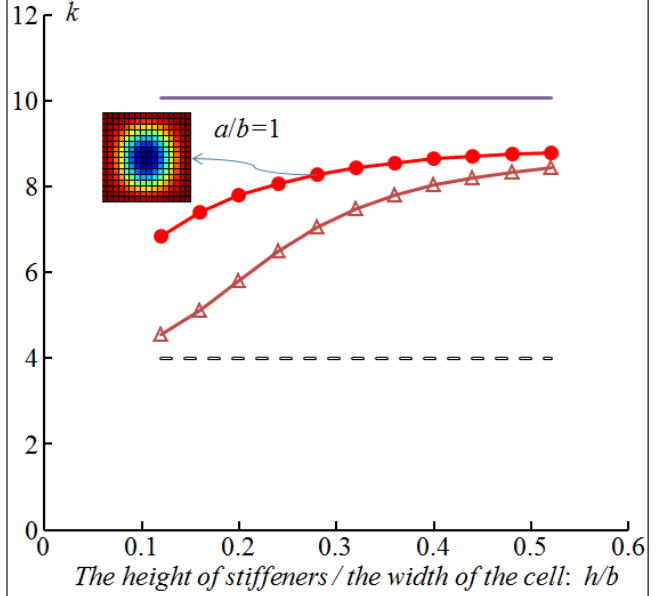

(b) $a / b=1$

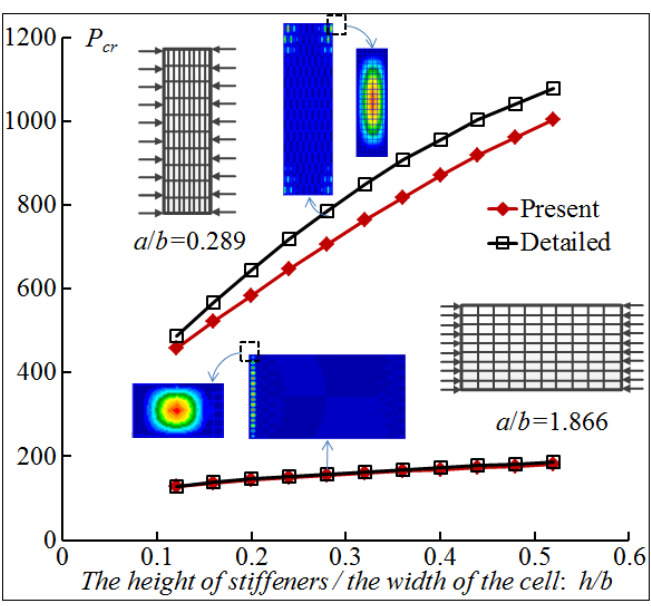

(d) Validate the proposed method by the detailed finite element model

Figure 4: A comparison between the skin local buckling predicted by the proposed method and the semi-analytical method of a flat orthogrid-stiffened isotropic panel under axial compression.

In practice, there is no evident local buckling for a grid-stiffened panel with a small height of stiffeners. Numerically, there is no local minimum in the critical buckling load parameter 
surface detected for ratios of the stiffener height and the length of the panel less than a specific value. Investigations of the local buckling in this example are done for cell configurations with the height of the stiffeners changing from $0.12 b$ to $0.52 b$ and the width of the stiffener fixed to be $0.02 b$. All the stiffeners are eccentrically located. Three typical aspect ratios $a / b$ of 1.866, 1 and 0.289 are considered for the cell configuration in accordance with the reference [18]. As illustrated in Fig. 4, the semi-analytical methods with periodic boundary conditions and with clamped boundary conditions for orthogrid-stiffened panels under axial compression always provide the lower bound and the upper bound of the skin buckling load coefficients predicted by the proposed method, respectively. In other words, the rectangular skin field under axial compression with the onset of local buckling predicted by the proposed method is always constrained with a boundary condition between simply supported and clamped, which can be concluded from both the values of the buckling load coefficients and the shape of the buckling modes.

A similar conclusion is drawn for the isogrid-stiffened panel, as illustrated in Fig. 5. It can be seen that results with the consideration of the interaction between adjacent skin fields provide a closer lower bound of the results predicted by the proposed method than those of simply supported boundary conditions. The torsional rigidity of the stiffeners ignored in the semi-analytical method takes a responsibility of the difference between results of the proposed method and the semi-analytical method, which is proved by the results with $G J=0$.

As illustrated in Fig.s 4(d) and 5(d), local buckling loads of the orthogrid- and isogridstiffened panels under axial compression predicted by the proposed method are validated by the results of corresponding finite element models with detailed stiffeners for cell configurations with two extreme aspect ratios. The largest errors for the sampled orthogrid-stiffened panels with aspect ratios of 1.866 and 0.289 are $-3 \%$ and $-10 \%$, respectively, while they are $20 \%$ and $8 \%$ for the sampled isogrid-stiffened panels. These errors are acceptable in practice. Moreover, the buckling modes obtained using the proposed method agree with those in a cell configuration of the detailed finite element models. All the detailed finite element models in this example employ a lattice with 16 cells in the width direction and 16 cells in the height direction and the particular geometric and material parameters are as follows: the width of the cell configuration $b=100 \mathrm{~mm}$, the skin thickness $t=1 \mathrm{~mm}$, the width of the stiffeners $w=2 \mathrm{~mm}$, the Young's modulus $E=206.84 \mathrm{GPa}$ and the Poisson's ratio $v=1 / 3$.

From the comparisons, it can be concluded that the proposed method has the ability to predict the local buckling load of orthogrid- and isogrid-stiffened structures with stiffeners of different rigidities and the prediction results are more accurate than those calculated by the semi-analytical method with periodic boundary conditions, which have already been improved compared with those with simply supported boundary conditions. 


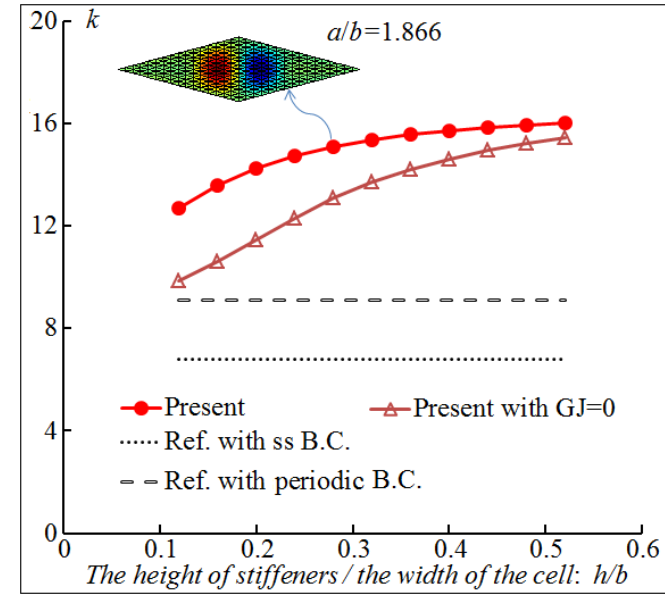

(a) $a / b=1.866$

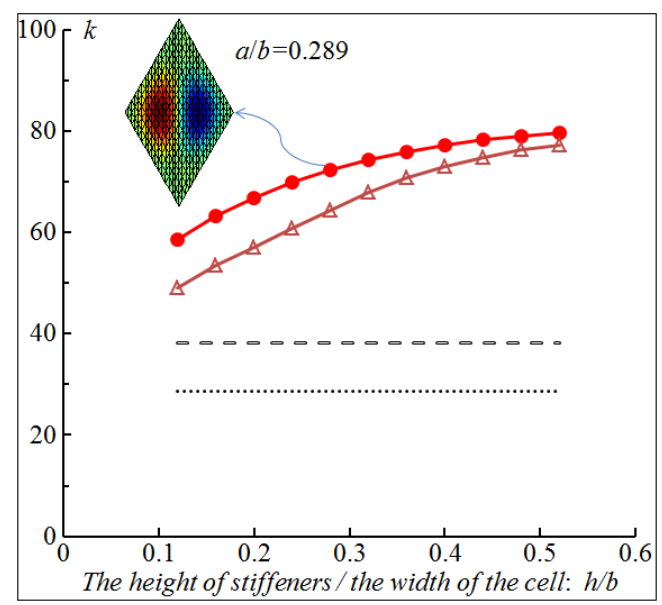

(c) $a / b=0.289$

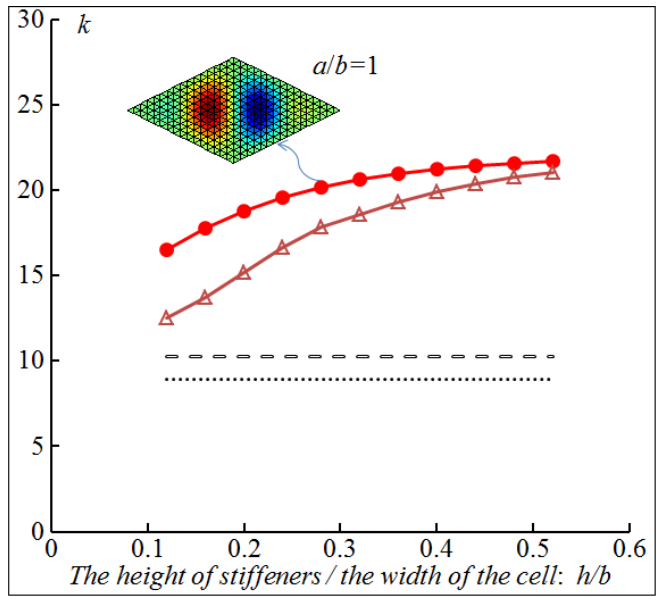

(b) $a / b=1$

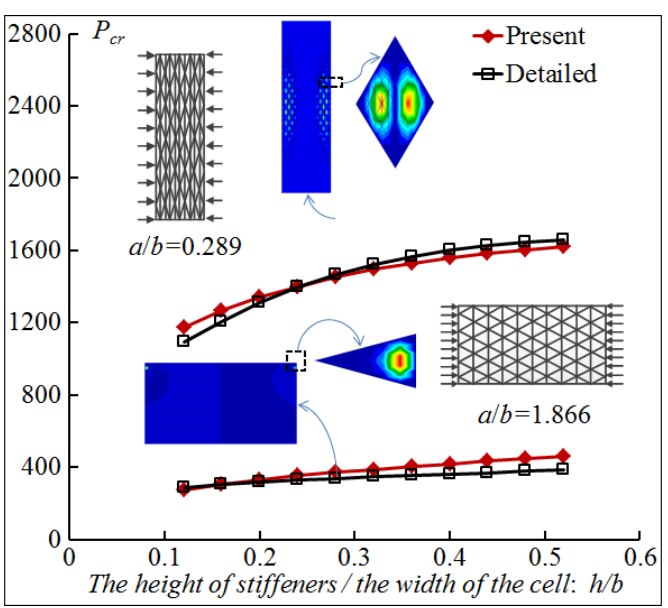

(d) Validate the proposed method by the detailed finite element model

Figure 5: A comparison between the skin local buckling predicted by the proposed method and the semi-analytical method of a flat isogrid-stiffened isotropic panel under axial compression.

\subsection{A flat composite panel}

The example is a simply supported flat grid-stiffened composite panel, which represents a generic structural component of a transport helicopter fuselage, as studied in reference [20]. The flat panel with the length of $4376.4 \mathrm{~mm}$ and the width of $1587.7 \mathrm{~mm}$ is stiffened on one side by orthogrid or isogrid stiffeners. The skin laminate has an eight-plys symmetric layup of $( \pm 45 / 90 / 0)_{s}$ with each ply thickness being $0.1524 \mathrm{~mm}$. The nominal ply mechanical properties are $E_{11}=139.31 \mathrm{GPa}, E_{22}=13.103 \mathrm{GPa}, G_{12}=G_{13}=G_{23}=5.0345 \mathrm{GPa}, v_{12}=0.3$ and $\rho=1590 \mathrm{~kg} / \mathrm{mm}^{3}$. All the stiffeners are made of 0 -deg material with a height of $h=12.9 \mathrm{~mm}$ and a width of $w=1.524 \mathrm{~mm}$.

The parameters of the grid-stiffened flat panel are given in Table 1. In the example, the angles 
between different stiffeners are set to be $60^{\circ}$ for the isogrid cell configurations. The weights of the involved orthogrid- and isogrid-stiffened flat panels are very close.

Table 1: Parameters of a grid-stiffened flat panel.

\begin{tabular}{|c|c|c|c|c|}
\hline \multirow{2}{*}{ Grid type } & \multirow{2}{*}{$\begin{array}{l}\text { Dimensions of a } \\
\text { cell: } s_{1} / s_{2}(\mathrm{~mm})\end{array}$} & \multicolumn{2}{|c|}{ No. of stiffeners } & \multirow{2}{*}{$\begin{array}{c}\text { Weight of } \\
\text { stiffeners }(\mathrm{kg})\end{array}$} \\
\hline & & Vertical & Horizontal & \\
\hline Orthogrid & $109.41 / 63.508$ & 41 & 26 & 5.5917 \\
\hline Isogrid & 136.58 & 38 & $\backslash$ & 5.5586 \\
\hline
\end{tabular}

Table 2: Critical buckling loads of a grid-stiffened flat composite panel under compression.

\begin{tabular}{|c|c|c|c|c|}
\hline & Buckling & Proposed method & Detailed model & Error \\
\hline \multirow[t]{3}{*}{ Orthogrid } & Global & & & $-0.2 \%$ \\
\hline & Local & 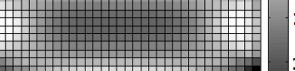 & & $-16.8 \%$ \\
\hline & & 126.61 & 152.15 & \\
\hline \multirow[t]{3}{*}{ Isogrid } & Global & & & $4.5 \%$ \\
\hline & Local & & & $12.3 \%$ \\
\hline & & 69.15 & 61.56 & \\
\hline
\end{tabular}

To investigate the critical buckling loads, a unit uniaxial compression of $1 \mathrm{~N} / \mathrm{mm}$ is considered. Both global and local buckling loads are given in Table 2. Local buckling behaviours predicted by the proposed method in Table 2 represents the distribution of local buckling loads. Compared with results of the detailed finite element model, global and local buckling loads predicted by the proposed method are within errors of $5 \%$ and $20 \%$, respectively, for the involved grid-stiffened panels. 


\subsection{A composite circular cylinder}

An orthogrid-stiffened composite circular cylinder with the diameter of $990 \mathrm{~mm}$ and the height of $1168 \mathrm{~mm}$ [6] is employed to investigate the critical buckling states of a cylinder under axial compression loading. The skin is made of AS4/3502 graphite/epoxy, while stiffeners are combinations of both AS4/3502 graphite/epoxy and filler. The mechanical properties of the skin and stiffeners are given in Table 3.

Table 3: Mechanical properties of grid-stiffened circular cylinders.

\begin{tabular}{ccc}
\hline & Skin & Stiffener \\
\hline$E_{11}: G P a$ & 127.5 & 64.86 \\
$E_{22}: G P a$ & 11.3 & 3.74 \\
$G_{12}: G P a$ & 6 & 0.93 \\
$v_{12}$ & 0.3 & 0.3 \\
\hline
\end{tabular}

The cylinder with a skin thickness of $1.22 \mathrm{~mm}$ suffers from an axial compression of $175 \mathrm{~N} / \mathrm{mm}$. To ensure the uniform distribution of structural forces, clamped boundary conditions are used with radial displacements not fixed but coupled to deform together for the top and bottom nodes, respectively. Two sets of dimensions of orthogrid-stiffened configurations are used corresponding to two different symmetric skin layups of $(0,90)_{s}$ and $( \pm 45)_{s}$, as shown in Table 4.

Table 4: Dimensions of grid-stiffened circular cylinders.

\begin{tabular}{ccccc}
\hline \multirow{2}{*}{ Skin layup } & \multirow{2}{*}{$\begin{array}{c}\text { No. of axial } \\
\text { stiffeners/rings }\end{array}$} & \multicolumn{2}{c}{ stiffeners: $m m$} \\
\cline { 3 - 5 } & & Height & Width \\
\hline \multirow{2}{*}{ Orthogrid } & $(0,90)_{s}$ & $90 / 20$ & 6.35 & 1.7 \\
& $( \pm 45)_{s}$ & $80 / 26$ & 3.175 & 1.81 \\
\hline
\end{tabular}

Stability analysis of the involved composite circular cylinders is carried out using the proposed method. Both global and critical local buckling loads of these cylinders are calculated and the results are provided in Table 5. The critical buckling load parameter surfaces with respect to dimensionless wave numbers are illustrated in items of the tables corresponding to local buckling behaviours obtained by the proposed method, where the local minimum represents the local buckling load.

As illustrated in Table 5, the errors of the global and local buckling loads using the proposed method are within $5 \%$ and $15 \%$, respectively, compared with results of the detailed finite element model. Noticeably, in this case, the global buckling modes obtained by the proposed method are not exactly the same as those by the detailed finite element model. For cylinder buckling, the first few global buckling modes are different but their buckling loads are very close. These modes may happen at a different sequence for the proposed method and the detailed finite element method, which results in the difference between the critical global buckling 
modes. For the orthogrid-stiffened cylinder with a skin layup of $( \pm 45)_{s}$, local buckling does not exist since there is no local minimum in the critical buckling load parameter surface with respect to dimensionless wave numbers, as illustrated in Table 5. Actually, the local buckling is trivial in this case since the global bucking dominates the failure mode completely.

Table 5: Critical buckling loads of the orthogrid-stiffened circular cylinder.

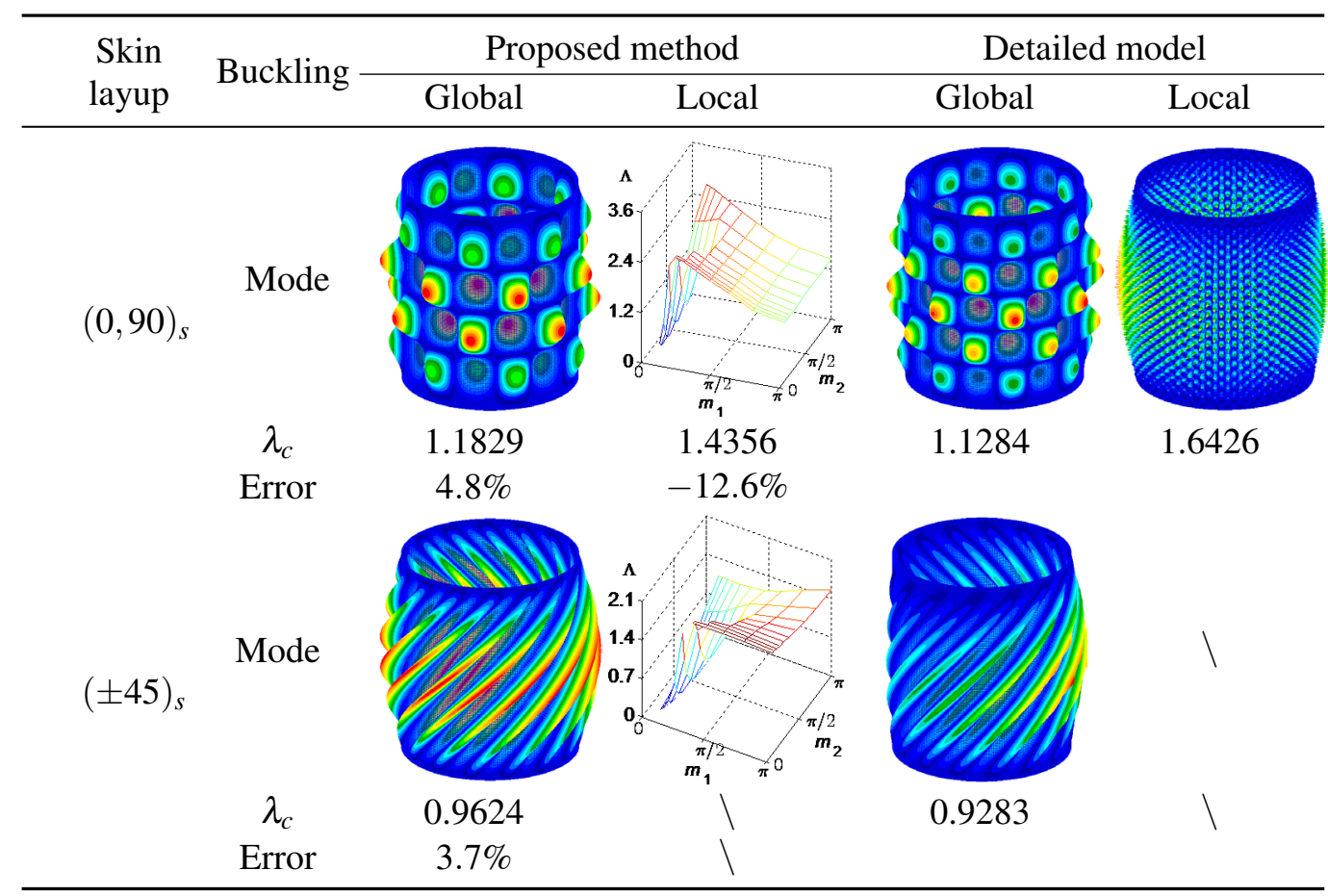

\section{Conclusions}

In this paper, buckling analysis of periodically grid-stiffened composite plates and shells is studied. Both global and local buckling phenomena are taken into consideration. A smearing method based on homogenization theory is employed to calculate the equivalent material properties. Global buckling loads are obtained by buckling analysis of the smeared model. Local buckling loads are predicted by the Bloch wave theory. In contrast to applications of the Bloch wave theory in periodic solid to investigate in-plane material failure, the paper concentrates on local buckling of grid-stiffened structures where the critical load always goes to zero as the wave length is increased. Thus, the local minimum in dimensionless wave number space is considered to be the critical local buckling load. Because of the used detailed finite element model of the cell, with the skin and stiffeners assembled together, both the skin buckling and stiffener crippling are possible critical modes. There is no need for separate stability analysis of the stiffener and the skin. It is concluded from the comparisons between local buckling load coefficients of the proposed method and the semi-analytical method that the proposed method could predict 
the local buckling load more accurately than the semi-analytical method using periodic boundary conditions, while the latter always provide a lower bound of the former. Representative numerical examples of grid-stiffened flat composite panels and circular composite cylinders are employed to prove the effectiveness of the proposed method. Comparison with the results of the detailed finite element model indicates that local buckling loads predicted by the proposed method have an error within $20 \%$ for compression loading.

\section{REFERENCES}

[1] Higgins, P.E.J., Wegner, P., Viisoreanu, A. and Sanford, G. E. Design and testing of the minotaur advanced grid-stiffened fairing. Compos. Struct. (2004) 66:339-349.

[2] Huybrechts, S.M. and Hahn, S.E. and Meink, T.E. E. Grid stiffened structures: a survey of fabrication, analysis and design methods. Proc. 12th Int. Conf. Compos. Mater. (ICCM/12), Paris, France, (1999).

[3] Jadhav, P. and Mantena, P. R. Parametric optimization of grid-stiffened composite panels for maximizing their performance under transverse loading. Compos. Struct. (2007) 77:353-363.

[4] Schraad, M. and Triantafyllidis, N. Scale effects in media with periodic and nearly periodic microstructures - I. Macroscopic properties. J. Appl. Mech. (1997) 64:751-762.

[5] Kassegne, S.K. and Reddy, J.N. Local behavior of discretely stiffened composite plates and cylindrical shells. Compos. Struct. (1998) 41:13-26.

[6] Reddy, A.D., Valisetty, R.R. and Rehfield, L.W. Continuous filament wound composite concepts for aircraft fuselage structures. J. Aircraft (1985) 22:249-255.

[7] Wang, J.T.S. and Hsu, T.M. Discrete analysis of stiffened composite cylindrical shells. AIAA J. (1985) 11:1753-1761.

[8] Wodesenbet, E., Kidane, S. and Pang, S.S. Optimization for buckling loads of grid stiffened composite panels. Compos. Struct. (2003) 60:159-169.

[9] Zhang, Z.F., Chen, H.R. and Ye, L. A stiffened plate element model for advanced grid stiffened composite plates/shells. J. Compos. Mater. (2011) 45:187-202.

[10] Hassani, B. and Hinton, E. A review of homogenization and topology optimization Ihomogenization theory for media with periodic structure. Comput. Struct. (1998) 69:707717.

[11] Michel, J.C., Moulinec, H. and Suquet, P. Effective properties of composite materials with periodic microstructure: a computational approach. Comput. Methods Appl. Mech. Eng. (1999) 172:109-143. 
[12] Caillerie, D. Thin elastic and periodic plates. Math. Methods Appl. Sci.) (1984) 6:159-191.

[13] Cecchi, A. and Sab, K. A homogenized Reissner-Mindlin model for orthotropic periodic plate: application to brickwork panels. Int. J. Solids Struct. (2007) 44:6055-6079.

[14] Lebée, A. and Sab, K. A bending-gradient model for thick plates. Part I: Theory. Int. J. Solids Struct. (2011) 48:2878-2888.

[15] Lebée, A. and Sab, K. A bending-gradient model for thick plates. Part II: Closed-form solutions for cylindrical bending of laminates. Int. J. Solids Struct. (2011) 48:2889-2901.

[16] Lebée, A. and Sab, K. Homogenization of thick periodic plates: application of the Bending-Gradient plate theory to a folded core sandwich panel. Int. J. Solids Struct. (2012) 49:2778-2792.

[17] Lebée, A. and Sab, K. Homogenization of a space frame as a thick plate: application of the Bending-Gradient plate theory to a beam lattice. Comput. Struct. (2013) 127:88-101.

[18] Weber, M.J. and Middendorf, P. Semi-analytical skin buckling of curved orthotropic gridstiffened shells. Compos. Struct. (2014) 108:616-624.

[19] Jaunky, N., Knight, Jr., N.F. and Ambur, D.R. Optimal design of general stiffened composite circular cylinders for global buckling with strength constraints. Compos. Struct. (1998) 41:243-252.

[20] Jaunky, N., Knight, Jr., N.F. and Ambur, D.R. Optimal design of grid-stiffened composite panels using global and local buckling analyses. J. Aircraft (1998) 35:478-486.

[21] Jaunky, N., Knight, Jr., N.F. and Ambur, D.R. Formulation of an improved smeared stiffener theory for buckling analysis of grid-stiffened composite panels. Compos. Part B Eng. (1996) 27B:519-526.

[22] Ambur, D.R. and Jaunky, N. Optimal design of grid-stiffened panels and shells with variable curvature. Compos. Struct. (2001) 52:173-180.

[23] Phillips, J.L. and Gürdal, Z. Analysis and optimum design of geodesically stiffened composite panels. Compos. Mater. Des. Anal. WIT Press, Southampton, pp. 509-528, (1990).

[24] Chen, H.J. and Tsai, S.W. Analysis and optimum design of composite grid structures. J. Compos. Mater. (1995) 30:503-533.

[25] Mead, D.J. Free wave propagation in periodically supported infinite beams. J. Sound Vib. (1970) 11:181-197.

[26] Mead, D.J. A new method of analyzing wave propagation in periodic structures; applications to periodic Timoshenko beams and stiffened plates. J. Sound Vib. (1986) 104:9-27. 
[27] Geymonat, G., Müller, S. and Triantafyllidis, N. Homogenization of nonlinearly elastic materials, microscopic bifurcation and macroscopic loss of rank-one convexity. Arch. Ration. Mech. Anal. (1993) 122:231-291.

[28] Triantafyllidis, N. and Schnaidt, W.C. Comparison of microscopic and macroscopic instabilities in a class of two-dimensional periodic composites. J. Mech. Phys. Solids (1993) 41:1533-1565.

[29] Triantafyllidis, N. and Bardenhagen, S. The influence of scale size on the stability of periodic solids and the role of associated higher order gradient continuum models. J. Mech. Phys. Solids (1996) 44:1891-1928.

[30] Schraad, M. and Triantafyllidis, N. Scale effects in media with periodic and nearly periodic microstructures - II. Failure mechanisms. J. Appl. Mech. (1997) 64:763-771.

[31] Gong, L., Kyriakides, S. and Triantafyllidis, N. On the stability of Kelvin cell foams under compressive loads. J. Mech. Phys. Solids (2005) 53:771-794.

[32] Ohno, N., Okumura, D. and Niikawa, T. Long-wave buckling of elastic square honeycombs subject to in-plane biaxial compression. Int. J. Mech. Sci. (2004) 46:1697-1713.

[33] Okumura, D., Okada, A. and Ohno, N. Buckling behavior of Kelvin open-cell foams under [0 $\left.0 \begin{array}{ll}0 & 1\end{array}\right],\left[\begin{array}{lll}0 & 1 & 1\end{array}\right]$ and [ $\left[\begin{array}{lll}1 & 1 & 1\end{array}\right]$ compressive loads. Int. J. Solids Struct. (2008) 45:3807-3820.

[34] Triantafyllidis, N., Nestorović, M.D. and Schraad, M.W. Failure surfaces for finitely strained two-phase periodic solids under general in-plane loading. J. Appl. Mech. (2006) 73:505-515.

[35] Ohno, N., Okumura, D. and Noguchi, H. Microscopic symmetric bifurcation condition of cellular solids based on a homogenization theory of finite deformation. J. Mech. Phys. Solids (2002) 50:1125-1153.

[36] Galambos, T.V. Guide to stability design criteria for metal structures. John Wiley \& Sons, INC, 5th ed. (1998). 